\title{
Correlation Inequalities in Function Spaces
}

\author{
R. Ahlswede and V. Blinovsky
}

\begin{abstract}
We give a condition for a Borel measure on $R^{[0,1]}$ which is sufficient for the validity of an AD-type correlation inequality in the function space ${ }^{1}$
\end{abstract}

In [1] was proved that if $\varphi_{1}, \varphi_{2}, \varphi_{3}, \varphi_{4}$ are bounded real non negative measurable functions on the space with measure $\left(R^{n}, \mathcal{B}, \mu\right)$ which satisfy for all $\bar{x}, \bar{y} \in R^{n}$ the following inequality

$$
\varphi_{1}(\bar{x}) \varphi_{2}(\bar{y}) \leq \varphi_{3}(\bar{x} \bigvee \bar{y}) \varphi_{4}(\bar{x} \bigwedge \bar{y}) \text { a.s. },
$$

then

$$
\int \varphi_{1}(\bar{x}) \mu(d \bar{x}) \int \varphi_{2}(\bar{x}) \mu(d \bar{x}) \leq \int \varphi_{3}(\bar{x}) \mu(d \bar{x}) \int \varphi_{4}(\bar{x}) \mu(d \bar{x}),
$$

where $\mu(d \bar{x})$ is the product $\sigma$-finite measure on $\mathcal{B},(\bar{x} \vee \bar{y})_{i}=x_{i} \vee y_{i},(\bar{x} \wedge \bar{y})_{i}=$ $x_{i} \wedge y_{i}$. That proof was simplified in [2] via induction on dimension $n$ suggested in [3], [7] , [8]. The question we consider here is how the problem can be viewed in the case of not arbitrary measure $\nu$ on $R^{T}$, when possibly $T=[0,1]$ ? The next theorem answers this question.

Let for arbitrary real functions $x(t), y(t), t \in T=[0,1],(x \bigvee y)(t)=x(t) \bigvee y(t)$ and $(x \bigwedge y)(t)=x(t) \bigwedge y(t)$. Let also $\nu_{i}(d \bar{x}), i=1,2,3,4$ be measures on the Borel sets $\mathcal{B}(\mathcal{C})$ of the linear space $\mathcal{C}$ of continuous functions from $R^{T}$ with the norm $\|\cdot\|_{\infty}$, which are finite on the compact subsets of $\mathcal{C}$. Let also $\varphi_{i}, i=1,2,3,4$ be four uniformly bounded nonnegative Borel real functions from $R^{T}$.

Theorem 1. If the following conditions are valid

$$
\begin{aligned}
& \varphi_{1}(f) \varphi_{2}(g) \leq \varphi_{3}(f \bigvee g) \varphi_{4}(f \bigwedge g) \\
& \nu_{1}(A) \nu_{2}(B) \leq \nu_{3}(A \bigvee B) \nu_{4}(A \bigwedge B), A, B \in \mathcal{B}(\mathcal{C}),
\end{aligned}
$$

then

$$
\int \varphi_{1}(\bar{x}) \nu_{1}(d \bar{x}) \int \varphi_{2}(\bar{x}) \nu_{2}(d \bar{x}) \leq \int \varphi_{3}(\bar{x}) \nu_{3}(d \bar{x}) \int \varphi_{4}(\bar{x}) \nu_{4}(d \bar{x}) .
$$

Here $A \bigvee B=\{a \bigvee b: a \in A, b \in B\}, A \bigwedge B=\{a \bigwedge b: a \in A, b \in B\}$

Condition (4) is also necessary for (5). Indeed indicator functions $I_{A}, I_{B}, I_{A \vee B}$, $I_{A \wedge B}$ satisfy (3) and substitution of them in (5) gives (4).

\footnotetext{
${ }_{1}^{1}$ This work is partially supported by RFFI grants No 03-01-00592 and 03-01-00098 and INTAS grant No 00-738.
} 
We call a measure $\nu$ which satisfies the relation

$$
\nu(A) \nu(B) \leq \nu(A \bigvee B) \nu(A \bigwedge B), A, B \in \mathcal{B}(\mathcal{C})
$$

an FKG measure.

Proof. If

$$
\int \varphi_{3}(\bar{x}) \nu_{3}(d \bar{x}) \int \varphi_{4}(\bar{x}) \nu_{4}(d \bar{x})=\infty
$$

then (5) follows. Next we consider that

$$
\int \varphi_{3}(\bar{x}) \nu_{3}(d \bar{x}) \int \varphi_{4}(\bar{x}) \nu_{4}(d \bar{x})<\infty
$$

and propose at first that

$$
\int \varphi_{1}(\bar{x}) \nu_{1}(d \bar{x}) \int \varphi_{2}(\bar{x}) \nu_{2}(d \bar{x})<\infty .
$$

Then $\int \varphi_{i}(\bar{x}) \nu_{i}(d \bar{x}), i=1,2,3,4$ are finite Borel measures which are regular and hence there exists a compact set $\mathcal{K} \subset \mathcal{C}$, such that for given $\epsilon>0$

$$
\left|\int_{\mathcal{K}} \varphi_{i}(\bar{x}) \nu_{i}(d \bar{x})-\int \varphi_{i}(\bar{x}) \nu_{i}(d \bar{x})\right| \leq \epsilon, i=1,2,3,4
$$

and $\nu(\mathcal{K})<\infty$. This compact set is by Ascoli's Lemma the set of equicontinuous functions $\left\{x_{t}\right\}$ which for some $N>0$ satisfy the relation

$$
\left|x_{t}\right| \leq N
$$

Without loss of generality we will consider that $\mathcal{K}$ is the set of all such functions. It is easy to see that this set is a distributive lattice. Indeed if

$$
\left|x_{i}(t)-y(t)\right|<\epsilon,\left|x_{i}\right| \leq N, i=1,2
$$

then

$$
\begin{aligned}
& \left|\left(x_{1} \bigvee x_{2}\right)(t)-y(t)\right|<\epsilon,\left|\left(x_{1} \bigwedge x_{2}\right)(t)-y(t)\right|<\epsilon, \\
& \left|\left(x_{1} \bigvee x_{2}\right)(t)\right|<N,\left|\left(x_{1} \bigwedge x_{2}\right)(t)\right| \leq N
\end{aligned}
$$

We consider the partition of the interval $T$ into $m$ consecutive subintervals $\Delta_{i}=$ $\left[t_{i-1}, t_{i}\right), i=1,2, \ldots m-1, t_{0}=0, \Delta_{m}=\left[t_{m-1}, 1\right]$ of equal length choosing $m$ in such a way that if $t, t^{\prime} \in \Delta_{i}$, then

$$
\left|x_{t}-x_{t^{\prime}}\right|<\delta / 2
$$

Without loss of generality we assume that $N$ is integer and that $\delta=L^{-1}$ for some natural $L$. Next we divide the interval $[-N, N]$ into $2 N / \delta=2 L N$ consecutive subintervals $\Gamma_{j}=\left[s_{j-1}, s_{j}\right), j=1,2, \ldots, 2 L N-1, \Gamma_{2 L N}=\left[s_{2 L N-1}, 2 L N\right]$ of equal length $\delta$. At last we consider the partition of the compact $\mathcal{K}$ into the set of cylinders $(m=2 L N+1)$

$$
\pi_{t_{0}, t_{1}, \ldots, t_{m}}\left(i_{0}, i_{1}, \ldots, i_{m}\right)=\left\{x_{t}: x_{t_{j}} \in \Gamma_{i_{j}}\right\}, i_{j}=1,2, \ldots, 2 L N
$$


Consider the finite set of rectangles $K\left(i_{0}, i_{1}, \ldots, i_{m}\right) \triangleq K_{t_{0}, t_{1}, \ldots, t_{m}}\left(i_{0}, i_{1}, \ldots, i_{m}\right)=\left\{x_{t}: x_{t_{j}} \in \Gamma_{i_{j}} ;\left|x_{t}-y_{j}\right|<\delta, t \in \Delta_{j}\right\} \subset R^{T}$, where $y_{j}$ is the center of the interval $\Gamma_{j}$. Then from (9) it follows that

$$
\mathcal{K} \subset \bigcup_{i_{j}} K_{t_{0}, t_{1}, \ldots, t_{m}}\left(i_{0}, i_{1}, \ldots, i_{m}\right) .
$$

Note also that

$$
\operatorname{diam}\left(K\left(i_{0}, i_{1}, \ldots, i_{m}\right)\right)=2 \delta .
$$

Now we approximate in $L^{1}\left(R^{T}, \nu_{i}\right)$ functions $\varphi_{i}$ on the compact $\mathcal{K}$ by continuous functions $f_{i}$ on $\mathcal{K}$ :

$$
\int_{\mathcal{K}}\left|\varphi_{i}(\bar{x})-f_{i}(\bar{x})\right| \nu_{i}(d \bar{x})<\epsilon .
$$

Using a standard procedure we can choose $f_{i}$ in such a way that

$$
f_{i} \leq \varphi_{i}, i=1,2 ; f_{i} \geq \varphi_{i}, i=3,4 .
$$

Note, that functions $f_{i}$ are uniformly continuous on $\mathcal{K}$ and consequently, choosing $\delta$ sufficiently small, we can choose new functions $\xi_{i}, i=1,2,3,4$ on $\mathcal{K}$ such that $0 \leq f_{i}-\xi_{i}<\epsilon / \nu_{i}(\mathcal{K}), i=1,2 ; 0 \leq \xi_{i}-f_{i}<\epsilon / \nu_{i}(\mathcal{K}), i=3,4$ and every $\xi_{i}$ is constant on every set $K_{t_{0}, t_{1}, \ldots, t_{m}}\left(i_{0}, i_{1}, \ldots, i_{m}\right) \cap \mathcal{K}$. At last note that the family $\mathcal{A}$ of sets

$$
K\left(i_{0}, i_{1}, \ldots, i_{m}\right) \bigcap \mathcal{K}, i_{j}=1,2, \ldots, 2 L N
$$

is a distributive lattice under the operations $\bigvee, \wedge$ on the set of indices $i_{j}$ :

$$
\begin{aligned}
& K\left(i_{0} \bigvee i_{0}^{\prime}, i_{1} \bigvee i_{1}^{\prime}, \ldots, i_{m} \bigvee i_{m}^{\prime}\right) \bigcap \mathcal{K} \\
& K\left(i_{0} \bigwedge i_{0}^{\prime}, i_{1} \bigwedge i_{1}^{\prime}, \ldots, i_{m} \bigwedge i_{m}^{\prime}\right) \bigcap \mathcal{K} \in \mathcal{A}
\end{aligned}
$$

Hence we have eight families of values

$$
\begin{aligned}
& \nu_{i}\left(i_{0}, i_{1}, \ldots, i_{m}\right) \triangleq \nu_{i}\left(K\left(i_{0}, i_{1}, \ldots, i_{m}\right)\right), i=1,2,3,4, \\
& \xi_{i}\left(i_{0}, i_{1}, \ldots, i_{m}\right) \triangleq \xi_{i}(\bar{x}), \bar{x} \in K\left(i_{0}, i_{1}, \ldots, i_{m}\right)
\end{aligned}
$$

and

$$
\begin{aligned}
& \nu_{1}\left(i_{0}, i_{1}, \ldots, i_{m}\right) \xi_{1}\left(i_{0}, i_{1}, \ldots, i_{m}\right) \nu_{2}\left(j_{0}, j_{1}, \ldots, j_{m}\right) \xi_{2}\left(j_{0}, j_{1}, \ldots, j_{m}\right) \leq(12) \\
\leq & \nu_{3}\left(i_{0} \bigvee j_{0}, i_{1} \bigvee j_{1}, \ldots, i_{m} \bigvee j_{m}\right) \xi_{3}\left(i_{0} \bigvee j_{0}, i_{1} \bigvee j_{2}, \ldots, i_{m} \bigvee j_{m}\right) \times \\
\times & \nu_{4}\left(i_{0} \bigwedge j_{0}, i_{1} \bigwedge j_{1}, \ldots, i_{m} \bigwedge j_{m}\right) \xi_{4}\left(i_{0} \bigwedge j_{0}, i_{1} \bigwedge j_{1}, \ldots, i_{m} \bigwedge j_{m}\right)
\end{aligned}
$$

Hence we are in the condition (1), (2) with counting measure $\mu$ on $R^{m+1}$

$$
\mu(A)=\sum_{i_{j}} \delta_{\bar{x},\left(i_{0}, i_{1}, \ldots, i_{m}\right)}(A)
$$


and

$$
\varphi_{i}\left(i_{0}, i_{1}, \ldots, i_{m}\right)=\nu_{i}\left(i_{0}, i_{1}, \ldots, i_{m}\right) \xi_{i}\left(i_{0}, i_{1}, \ldots, i_{m}\right) .
$$

It follows that

$$
\begin{aligned}
& \sum_{i_{j}} \nu_{1}\left(i_{0}, i_{1}, \ldots, i_{m}\right) \xi_{1}\left(i_{0}, i_{1}, \ldots, i_{m}\right) \sum_{i_{j}} \nu_{2}\left(j_{0}, j_{1}, \ldots, j_{m}\right) \xi_{2}\left(j_{0}, j_{1}, \ldots, j_{m}\right) \\
\leq & \sum_{i_{j}} \nu_{3}\left(i_{0}, i_{1}, \ldots, i_{m}\right) \xi_{3}\left(i_{0}, i_{1}, \ldots, i_{m}\right) \sum_{i_{j}} \nu_{4}\left(i_{0}, i_{1}, \ldots, i_{m}\right) \xi_{4}\left(i_{0}, i_{1}, \ldots, i_{m}\right) .
\end{aligned}
$$

Because

$$
\left|\sum_{i_{j}} \nu_{i}\left(i_{0}, i_{1}, \ldots, i_{m}\right) \xi_{i}\left(i_{0}, i_{1}, \ldots, i_{m}\right)-\int \xi_{i}(\bar{x}) \nu_{i}(d \bar{x})\right| \leq 3 \epsilon
$$

and $\epsilon>0$ is arbitrary from (13) it follows statement of the theorem in the case when (6) is valid. Let's consider now that (6) is not valid. Let's for example

$$
\int \varphi_{3}(\bar{x}) \nu_{3}(d \bar{x})=\infty, \int \varphi_{4}(\bar{x}) \nu_{4}(d \bar{x})<\infty .
$$

Then we use the same consideration, but instead of relations (7), (11) we apply relations

$$
\begin{gathered}
\int_{\mathcal{K}} \varphi_{3}(\bar{x}) \nu_{3}(d \bar{x})>M, \\
\int_{\mathcal{K}} f_{3}(\bar{x}) \mid \nu_{3}(d \bar{x})>M-\epsilon, f_{3}<M_{1}
\end{gathered}
$$

correspondingly. Here $M, M_{1}$ are given constants which we will an consider arbitrary large. Repeating the proof as in the case of finite integrals we obtain that the product integrals over measures $\nu_{3}, \nu_{4}$ is arbitrary large which gives the contradiction to their finiteness. Cases other than (14) are considered similarly. This proves the theorem.

Let's show that (4) is valid if we consider the all equal for different $i$ measures, generated by Wiener process. Actually it is not difficult to see that for the validness of (4) it is necessary and sufficient the inequality (4) to be valid for the cylinders $A, B$ which bases are rectangles. It easily follows from the proof of Theorem.

Hence all we should do when the random process is given by its finite dimensional distributions is to check whether it has continuous modification and whether the measure on cylinders which bases are rectangles generated by the finite dimensional distributions satisfy the inequality (4).

It is easy to see that Wiener process is the case. Indeed it is well known that in finite dimensional case Gauss distribution generate the measure, satisfying (4) if $r_{i, j} \leq 0, i \neq j$, where $W=\left\|w_{i, j}\right\|_{i, j=1}^{n}$ is the matrix inverse to the correlation matrix $R[2]$. In the case of Wiener process $R=\left\|t_{i} \bigvee t_{j}\right\|_{i, j=1}^{n}, t_{i}>t_{j}, i>j$ and the inverse matrix $W$ has nonzero elements only on the diagonal and also elements in the strip above and belong the diagonal $w_{p, p+1}=w_{p+1, p}=\left(t_{p}-t_{p+1}\right)^{-1}$, 
$p=1,2, \ldots, n-1$. Hence measure $\nu$, generated by the Wiener process $\omega_{t}$ satisfies (4) $\left(\nu_{i}=\nu, i=1,2,3,4\right)$.

Also introduce one important example of the function which satisfies (3). Such function is

$$
\varphi_{i}(\omega .)=\varphi(\omega .)=\exp \left(\int_{0}^{1} b(t) \omega_{t} d t\right)
$$

where $b(t)$ is some function for which the integral has sense. This functions satisfy (3) because the expression in the exponent is linear function of $\omega_{t}$ and the obvious relation

$$
a+b=a \bigvee b+a \bigwedge b .
$$

Next we introduce some corollaries of the theorem. Note that if $\ell$ is a nondecreasing non negative function, then functions $\varphi_{1}=\varphi_{3}=\ell, \varphi_{2}=\varphi_{4}=1$ satisfy inequality (3). If $\nu_{i}$ are probability distributions then from the theorem it follows that

$$
E_{1}(\ell) \leq E_{2}(\ell)
$$

where $E_{i}$ is the mathematical expectation under the probability measure $\nu_{i}$. Note that the theorem is valid also if instead of $T=[0,1]$ one consider $T=$ $n$. In other words the theorem is valid in $n$-dimensional space. To see it is enough to make minor changes in the proof mostly concerning notations. But in this case we have the extending of the result from [3],[7],[8] to the case of the arbitrary measure, not only such that is discrete or have density. The same note is valid concerning the FKG inequality [6]. Note also that the condition that $\ell \geq 0$ is ambitious because if it is negative we can consider the truncated version of $\ell$ and add the positive constant and then take a limit when the level of the truncation tends to infinity. If we have probability measure which satisfies inequality (4) (all $\nu_{i}$ are equal) then for any pair of nondecreasing (non increasing) functions $\ell_{1}, \ell_{2}$ the following inequality is valid

$$
E\left(\ell_{1} \ell_{2}\right) \geq E\left(\ell_{1}\right) E\left(\ell_{2}\right) .
$$

In the case of finite dimension it is a strengthening of the FKG inequality for the case of an arbitrary probability measure. To prove this inequality it is sufficient to use inequality (15) with $\nu_{2}(d \bar{x})=\frac{\ell_{2} \nu(d \bar{x})}{E\left(\ell_{2}\right)}, \nu_{1}=\nu$ and assume that $\ell_{2}$ is non negative.

\section{References}

1. R. Ahlswede and D. Daykin, An inequality for weights of two families of sets, their unions and intersections, Z. Wahrscheinlichkeitstheorie und verw. Gebiete, 93, 183$185,1979$.

2. S. Karlin and Y. Rinott, Classes of orderings of measures and related correlation inequalities, I. Multivariate Totally Positive Distributions, Journal of Multivariate Analysis, 10, 467-498, 1980.

3. J. Kemperman, On the FKG inequality for measures in a partially ordered space, Indag.Math, 39, 313-331, 1977. 
4. G. Birkhoff, Lattice Theory, Providence, RI, 1967.

5. J. Doob, Stochastic Processes, New York, Wiley, 1967.

6. C. Fortuin, P. Kasteleyn, and J. Ginibre, Correlation inequalities on some partially ordered sets, Comm. Math. Phys. 22, 89-103, 1971.

7. R. Holley, Remarks on the FKG inequalities, Comm. Math. Phys. 36, 227-231, 1974.

8. C. Preston, A generalization of the FKG inequalities, Comm. Math. Phys. 36, 233$241,1974$. 